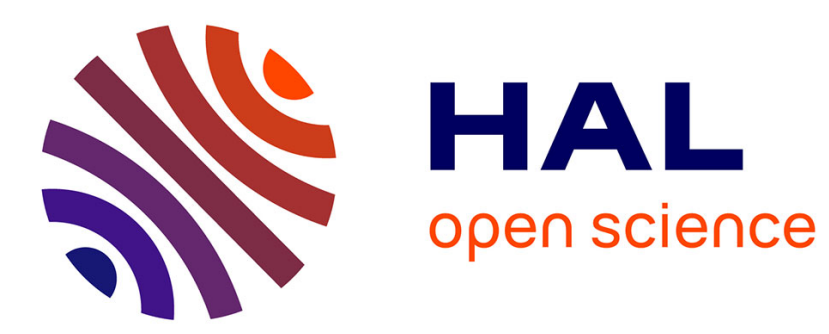

\title{
Handbook of bi-dimensional tensors: Part I: Harmonic decomposition and symmetry classes
}

Nicolas Auffray, Boris Kolev, Marc Olive

\section{To cite this version:}

Nicolas Auffray, Boris Kolev, Marc Olive. Handbook of bi-dimensional tensors: Part I: Harmonic decomposition and symmetry classes. Mathematics and Mechanics of Solids, 2017, 9 (22), pp.18471865. 10.1177/1081286516649017. hal-01303964

\section{HAL Id: hal-01303964 https://hal.science/hal-01303964}

Submitted on 29 May 2016

HAL is a multi-disciplinary open access archive for the deposit and dissemination of scientific research documents, whether they are published or not. The documents may come from teaching and research institutions in France or abroad, or from public or private research centers.
L'archive ouverte pluridisciplinaire HAL, est destinée au dépôt et à la diffusion de documents scientifiques de niveau recherche, publiés ou non, émanant des établissements d'enseignement et de recherche français ou étrangers, des laboratoires publics ou privés. 


\title{
Handbook of bidimensional tensors: \\ Part I: Harmonic decomposition and symmetry classes
}

Journal Title

$\mathrm{XX}(\mathrm{X}): 1-20$

(C)The Author(s) 2015

Reprints and permission:

sagepub.co.uk/journalsPermissions.nav

DOI: $10.1177 /$ ToBeAssigned

www.sagepub.com/

\author{
N. Auffray ${ }^{1}$ and B. Kolev ${ }^{2}$ and M. Olive ${ }^{3}$
}

\begin{abstract}
To investigate complex physical phenomena, bi-dimensional models are often an interesting option. It allows spatial couplings to produce while keeping them as simple as possible. For linear physical laws, constitutive equations involve the use of tensor spaces. As a consequence the different types of anisotropy that can be described are encoded in tensor spaces involved in the model. In the present paper, we solve the general problem of computing symmetry classes of constitutive tensors in $\mathbb{R}^{2}$ using mathematical tools coming from representation theory. The power of this method is illustrated through the tensor spaces of Mindlin strain-gradient elasticity.
\end{abstract}

\section{Keywords}

Anisotropy; Symmetry classes; Constitutive laws; Higher-order tensors

\section{Introduction}

To investigate complex physical phenomena such as anisotropic damage mechanics, elastic couplings within composite structures, or wave propagation in architected materials, bi-dimensional models constitute an interesting step before studying the complete 3D situation. It allows spatial couplings while keeping the process as simple as possible.

For linear physical laws, constitutive equations are described by tensors. A sound analysis of these tensors, and of the vectorial spaces they belong to, gives precious information on the physics that can be described by their mean. This way of exploring models through their tensorial description is well-developed in condensed matter physics [25], but not so much known in continuum mechanics. Nevertheless, these last years a renew of interest in tensor methods in the field of continuum mechanics has been observed [15, 14, 19]. This fact is related to the wish to properly design materials' architecture to optimize overall specific features. The spectrum of tensor methods is relatively broad and includes, among other, the optimal conception of composite materials [38], damage mechanics [20, 15], full-field identification method [7], and wave control [8, 13, 4, 32]. These methods are all based on the invariants of the elasticity tensor. These quantities allow to determine

\footnotetext{
${ }^{1}$ MSME, Université Paris-Est, Laboratoire Modélisation et Simulation Multi Echelle,MSME UMR 8208 CNRS, 5 bd Descartes, 77454 Marne-la-Vallée, France

${ }^{2}$ Aix Marseille Université, CNRS, Centrale Marseille, I2M, UMR 7373, 13453 Marseille, France

${ }^{3}$ LMA, CNRS UPR 7051, 13402 Marseille Cedex 20, France

Corresponding author:

N. Auffray, MSME, Université Paris-Est, Laboratoire Modélisation et Simulation Multi Echelle, MSME UMR 8208 CNRS, 5 bd Descartes, 77454 Marne-la-Vallée, France

Email: Nicolas.auffray@univ-mlv.fr
} 
an elastic material uniquely [11], and the polynomial relations between them indicate the symmetry classes of this material [5].

Tensor methods are not restricted to elasticity and can naturally be applied to other linear laws. For examples, the piezoelectricity effect is modelled by a third order tensor [21, 36], and extended models of elasticity involve tensors of order greater than $4[16,35]$. Hence, instead of treating each specific case separately, we found interesting to formulate the problem in its full generality to obtain general results.

In the following, tensor spaces used to model physical laws will be called Constitutive Tensor Spaces, a precise construction of those spaces will be given latter on. Considering a constitutive tensor space, from a physical perspective at least three questions naturally arise:

1. What are the different types of anisotropic behaviour that can be described ?

2. How can different materials (with respect to a chosen physics) be uniquely labelled ?

3. How to identify the anisotropic class of a specific tensor from its components in an arbitrary basis?

In this paper, attention will only be devoted to the first of these three questions and only in the $2 \mathrm{D}$ situation. The two other questions, which have been solved for elasticity in 2D by Vianello [37], will be the objects of forthcoming papers. Albeit being different in essence, those questions are closely related by the use of the same mathematical tools. Before getting into the core of the matter, let us give few words on the historical background of our problem.

\section{State of the arts}

The determination of the different type of anisotropy associated to a tensor space consists in computing the isotropy (or symmetry) classes of an $\mathrm{SO}(d)$ - or $\mathrm{O}(d)$ - representation on that space. This problem has been properly set up in mechanics for the first time by Forte and Vianello in 1996 [17]. In their paper, they provided the number and type of symmetry classes for 3D linear elasticity. Since then, other specific studies in 3D have been conducted, and answers have been provided for photo-elasticity [18], piezo-electricity [21], or flexo-elasticity [26]. In two recent publications $[29,30]$ a general and complete answer has been provided for any $3 \mathrm{D}$ constitutive tensor space. Aside from the 3D situation, some studies have been conducted in 2D. The 2D elasticity tensor has been studied in numerous publications [10, 23, 37, 14, 19]. The last cited reference provides a clear and comprehensive review of most of the work that have been done on plane elasticity tensors. This synthesis is important since a lot of different denominations for the same technique can be found in the literature [19]. The other plane physical behaviours investigated have been: piezo-electricity [36], Mindlin strain gradient elasticity [3, 4]. Here, instead of considering each case separately, we propose to set the problem in its full generality and to provide a general answer from which specific cases can be deduced. This paper intents to be as self-contained as possible and many examples are provided along the lines.

\section{Organisation}

The article is organised as follows: section 2 is devoted to the formulation of the problematic, and by its end our main results are given and some examples provided. The next two sections are devoted to the proofs of these results. In section 3 a decomposition of tensor spaces into irreducible components is introduced. This decomposition, also refereed to as the harmonic decomposition, is the cornerstone of our analysis of the geometrical properties of tensor spaces. In section 4, the link is established between harmonic decomposition and symmetry classes through the use of a tool named the clips operator [29, 30].

\section{Notations}

Let us introduce the main notations that will be used throughout the paper. The following matrix groups will be considered:

- GL(2): the group of all linear invertible transformations of $\mathbb{R}^{2}$, i.e. $\mathrm{F} \in \mathrm{GL}(2)$ iff $\operatorname{det}(\mathrm{F}) \neq 0$; 
- $\mathrm{O}(2)$ : the orthogonal group, that is the group of all isometries of $\mathbb{R}^{2}$ i.e. $\mathrm{Q} \in \mathrm{O}(2)$ iff $\mathrm{Q} \in \mathrm{GL}(2)$ and $\mathrm{Q}^{-1}=\mathrm{Q}^{T}$, where the superscript ${ }^{T}$ denotes the transposition. As a matrix group $\mathrm{O}(2)$ can be generated by:

$$
r_{\theta}=\left(\begin{array}{cc}
\cos \theta & -\sin \theta \\
\sin \theta & \cos \theta
\end{array}\right), 0 \leq \theta<2 \pi, \quad \text { and } \quad \sigma_{x}=\left(\begin{array}{cc}
1 & 0 \\
0 & -1
\end{array}\right)
$$

in which $r_{\theta}$ is a rotation by angle $\theta$ and $\sigma_{x}$ is the reflection across the $x$ axis;

- $\mathrm{SO}(2)$ : the special orthogonal group, i.e. the subgroup of $\mathrm{O}(2)$ consisting of transformations satisfying $\operatorname{det}(\mathrm{Q})=1$. It corresponds to the group of plane rotations generated by $r_{\theta}$;

Classical notations will be used to denote tensor products:

- $\otimes$ stands for the classical product, and $\otimes^{n}$ indicates its $n$-th power;

- $S^{2}$ denotes its symmetrized version, and $S^{n}$ its extension to product of $n$ elements;

- $\wedge^{2}$ indicates the antisymmetric tensor product.

Vector spaces will be denoted using blackboard fonts. The subsequent tensor spaces will be considered:

- $\mathbb{G}^{n}:=\otimes^{n} \mathbb{R}^{2}$ is the space of general $n$ th-order tensors, that is tensors with no particular index symmetry;

- $\mathbb{S}^{n}:=S^{n}\left(\mathbb{R}^{2}\right)$ is the subspace of totally symmetric tensors of $\mathbb{G}^{n}$;

- $\mathbb{T}^{n}$ is any subspace of $\mathbb{G}^{n}$ which contains $\mathbb{S}^{n}$; it is generated by taking repeated tensorial products $\otimes^{p}$ or $S^{q}$ of $\mathbb{R}^{2}$;

- $\mathbb{K}^{n}$ is the space of $n$-th order completely symmetric and traceless tensors on $\mathbb{R}^{2}$, called harmonic tensors (this space is described in subsection 3.1).

When needed, index symmetries of both spaces and their elements are expressed as follows: (..) indicates invariance under permutations of the indices in parentheses,... and $\div .$. indicate, respectively, symmetry and antisymmetry with respect to permutations of the underlined blocks.

\section{Bidimensional tensors}

In the following, the physical space will be described by $\mathcal{E}^{2}$, the 2-dimensional Euclidean space. The mathematical framework used in this section is introduced in Appendix A.

\subsection{Material and physical symmetry groups}

We represent a physical body as a compact 2-dimensional manifold $\mathcal{M}$ embedded in the Euclidean space $\mathcal{E}^{2}$ and having a microstructure attached to each of its points $P \in \mathcal{M}$. This microstructure describes the local organisation of matter at a scale below the one used for the continuous description. For crystalline materials this microstructure corresponds to the crystal lattice, for polymers to the organisation of polymeric chains, .... As for crystals, microstructures can possess invariance properties with respect to orthogonal transformations $\mathrm{Q} \in \mathrm{O}(2)$. Hence, at each material point $P$, the set of such transformations forms a point group $\mathrm{G}_{\mathcal{M}}(P) \subset \mathrm{O}(2)$ which describes local material symmetries. At the continuous macroscopic scale detailed description of the microstructure is lost, and information on the microstructure is contained in $\mathrm{G}_{\mathcal{M}}(P)$. In the case of an homogeneous medium the point dependence vanishes and $\mathrm{G}_{\mathcal{M}}(P)=\mathrm{G}_{\mathcal{M}}$.

In this paper our attention will be restricted to linear constitutive laws. In this perspective, physical properties are encoded by tensors: we attach a tensor $\mathrm{T}(P)$ to each point $P$ of $\mathcal{M}$, resulting in a tensor field $\mathrm{T}$ that needs not be continuous in general. If the material is homogeneous this tensor field is constant, and the material is thus described by a single tensor. In general, the physical model imposes some index symmetries and $\mathrm{T}(P)$ belongs to $\mathbb{T}^{n} \subset \mathbb{G}^{n}$. The action of $\mathrm{O}(2)$ on an element of $\mathbb{T}^{n}$ is defined by:

$$
(\mathrm{Q} \cdot \mathrm{T})_{i_{1} i_{2} \ldots i_{n}}:=\mathrm{Q}_{i_{1} j_{1}} \ldots \mathrm{Q}_{i_{n} j_{n}} \mathrm{~T}_{j_{1} \ldots j_{n}} .
$$

Prepared using sagej.cls 
Hence, within our hypotheses, the symmetry group of a physical property described by $\mathrm{T}$ is defined as the set of operations that leave this tensor invariant. The symmetry group of $\mathrm{T}(P)$ is:

$$
\mathrm{G}_{\mathrm{T}(P)}:=\{\mathrm{Q} \in \mathrm{O}(2) \mid \mathrm{Q} \cdot \mathrm{T}(P)=\mathrm{T}(P)\} .
$$

Since constitutive tensors are supposed to describe physical properties of the matter, their symmetry groups must be connected. This connection is stated by the Curie-Neumann's Principle [39]:

Principle 2.1. Curie-Neumann's Principle. At each material point $P \in \mathcal{M}$ with a physical property $\mathcal{P}$ described by $\mathrm{T}(P) \in \mathbb{T}^{n}$, every material symmetry is a physical symmetry, i.e.:

$$
\mathrm{G}_{\mathcal{M}}(P) \subset \mathrm{G}_{\mathrm{T}(P)} \text {. }
$$

It is important to emphasize that the notion of point group naturally contains information on the orientation of either the "microstructure" or the "physical property" attached to this point with respect to a reference frame.

\subsection{Physical symmetry classes}

The intrinsic characterization of the symmetry of a physical object has to be independent of its orientation in space. Hence, as proposed by [17], from both a physical and mathematical point of view, the relevant notion to characterize the physical symmetry of an object is the symmetry class of its related tensor. Recall that for a tensor $\mathrm{T} \in \mathbb{T}^{n}$, having $\mathrm{G}_{\mathrm{T}}$ as symmetry group, its symmetry class is defined as the set of all the subgroups of $\mathrm{O}(2)$ conjugate to $\mathrm{G}_{\mathrm{T}}$ :

$$
\left[\mathrm{G}_{\mathrm{T}}\right]=\left\{\mathrm{QG}_{\mathrm{T}} \mathrm{Q}^{T} \subset \mathrm{O}(2), \mathrm{Q} \in \mathrm{O}(2)\right\}
$$

In other words, the symmetry class of $\mathrm{T}$ corresponds to its symmetry group modulo its orientation in $\mathrm{O}(2)$. Furthermore, it is known that there is only a finite number of different symmetry classes [12], and that each symmetry group is a closed subgroup of $\mathrm{O}(2)$. Conjugacy classes of closed subgroup of $\mathrm{O}(2)$ are known [2] and can be described as follows:

$$
\left\{[1],\left[\mathrm{Z}_{2}^{\sigma_{x}}\right],\left[\mathrm{Z}_{k}\right],\left[\mathrm{D}_{k}\right],[\mathrm{SO}(2)],[\mathrm{O}(2)]\right\}_{k \geq 2},
$$

in which 1 stands for the trivial subgroup, $\mathrm{SO}(2)$ is the continuous rotation group and the other discrete groups are described below:

- $\mathrm{Z}_{2}^{\sigma_{x}}$ is the group generated by $\sigma_{x}$, which denotes a mirror through the $x$-axis;

- $\mathrm{Z}_{k}$ is the cyclic group ${ }^{1}$ with $k \geq 2$ elements generated by the rotation $r_{2 \pi / k}$;

- $\mathrm{D}_{k}(k \geq 2)$, the dihedral group with $2 k$ elements generated by $r_{2 \pi / k}$ and $\sigma_{x}$.

In the previous designation $k$ indicates the order of the generating rotation of angle $\frac{2 \pi}{k}$. Obviously applying $k$ times this generating rotation will lead to identity. As it will be important latter on, this number can either be odd or even, and only in the first case the group contains the inversion -1 . By convention we would say that a group has an infinite order of rotation if it contains $\mathrm{SO}(2)$ as a subgroup.

\subsection{Constitutive tensor spaces}

Following [29, 30], constitutive tensor spaces are constructed according to the following procedure:

- Given two physical objects described by tensor spaces $\mathbb{T}^{m_{1}}$ and $\mathbb{T}^{m_{2}}$, for which we have

$$
\mathbb{S}^{m_{i}} \subset \mathbb{T}^{m_{i}} \subset \mathbb{G}^{m_{i}}, \quad i=1,2,
$$

a linear constitutive law is given by an element of the tensor space

$$
\mathbb{T}^{n}=\mathcal{L}\left(\mathbb{T}^{m_{1}}, \mathbb{T}^{m_{2}}\right) \simeq \mathbb{T}^{m_{1}} \otimes \mathbb{T}^{m_{2}},
$$

which, by construction, satisfies

$$
\mathbb{S}^{n} \subset \mathbb{T}^{n} \subset \mathbb{G}^{n}
$$

Prepared using sagej.cls 
- These inclusions can be refined with additional hypotheses:

- for self-adjoint constitutive laws, we have $m_{1}=m_{2}=m$ and tensor spaces $\mathbb{T}^{n}$ are constructed from the symmetric product:

$$
\mathbb{T}^{n}:=S^{2}\left(\mathbb{T}^{m}\right) .
$$

The symmetric tensor product is a consequence of the existence of a potential. The space of elasticity tensors is an example of such a situation.

- for coupling laws, tensor spaces $\mathbb{T}^{n}$ are constructed from the standard product:

$$
\mathbb{T}^{n}:=\mathbb{T}^{m_{1}} \otimes \mathbb{T}^{m_{2}} .
$$

This situation happens for coupling tensors that appear in multiphysical models (for instance, for the piezoelectric tensor).

Finally, we have to consider 3 different cases:

- for self-adjoint constitutive laws:

$$
S^{2}\left(\mathbb{S}^{m}\right) \subset S^{2}\left(\mathbb{T}^{m}\right) \subset S^{2}\left(\mathbb{G}^{m}\right) ;
$$

- for coupling laws:

$$
\mathbb{S}^{m_{1}} \otimes \mathbb{S}^{m_{2}} \subset \mathbb{T}^{m_{1}} \otimes \mathbb{T}^{m_{2}} \subset \mathbb{G}^{m_{1}+m_{2}}
$$

with 2 cases, whether $m_{1}$ and $m_{2}$ have the same parity or not. The order of resulting tensor space is even in the first case, and odd in the second.

\subsection{General results}

Motivated by Herman's theorem [24], which limits the possibilities for the symmetry class of a tensor of given order, we introduce the following definition.

Definition 2.2. For any integer $p \geq 1$, we define

$$
\left\{\begin{array}{l}
\Im_{\max }^{2 p}:=\left\{\left[\mathrm{Z}_{2 k}\right],\left[\mathrm{D}_{2 k}\right]\right\}_{1 \leq k \leq p} \cup\{[\mathrm{SO}(2)],[\mathrm{O}(2)]\} \\
\mathfrak{I}_{\max }^{2 p+1}:=\left\{\left[\mathrm{Z}_{2 k+1}\right],\left[\mathrm{D}_{2 k+1}\right]\right\}_{0 \leq k \leq p} \cup\{[\mathrm{O}(2)]\}
\end{array} .\right.
$$

These two sets correspond respectively to the maximal theoretical set of symmetry classes that a tensor space of order $2 p$ (resp. $2 p+1$ ) can possess.

Remark 2.3. Let us consider a physical property described by an odd-order tensor in $2 \mathrm{D}$. If the material symmetry group contains an even-order or an infinite-order rotation, then the related tensor is the null tensor. Indeed, classes related to finite subgroups in $\mathfrak{I}_{\max }^{2 p+1}$ contain only odd-order rotations, and the only odd-order tensor having $[\mathrm{O}(2)]$ as symmetry class is the null tensor. We can also note that classes related to finite subgroups in $\mathfrak{I}_{\max }^{2 p}$ only contain even-order rotations.

The main results of the present paper are summed up in the following tables and their proof are postponed in subsection 4.4.

Theorem 2.4. Symmetry classes. For every integers $p>0$ and $n>0$

\section{Table 1}

\begin{tabular}{|c|c|}
\hline Type of tensor space & Symmetry classes \\
\hline $\mathbb{S}^{n}$ & c.f. Lemma 2.5 \\
\hline $\mathbb{G}^{2 p}$ & $\mathfrak{I}_{\max }^{2 p}$ \\
\hline $\mathbb{G}^{2 p+1}$ & $\mathfrak{I}_{\max }^{2 p+1} \backslash\left\{\left[\mathrm{Z}_{2 p+1}\right]\right\}$ \\
\hline
\end{tabular}

Furthermore we have

Prepared using sagej.cls 


\section{Table 2}

\begin{tabular}{|c|c|}
\hline Type of tensor space & Symmetry classes \\
\hline$S^{2}\left(\mathbb{T}^{n}\right), \mathbb{S}^{n} \subsetneq \mathbb{T}^{n}$ & $\mathfrak{I}_{\max }^{2 n}$ \\
\hline$S^{2}\left(\mathbb{S}^{n}\right), n>2$ & $\mathfrak{I}_{\max }^{2 n} \backslash\left\{\left[\mathrm{Z}_{2 n}\right],\left[\mathrm{Z}_{2 n-2}\right]\right\}$ \\
\hline $\mathbb{T}^{m_{1}} \otimes \mathbb{T}^{m_{2}}, m_{i}>0$ & $\left\{\begin{array}{l}\mathfrak{I}_{\max }^{m_{1}+m_{2}}, \quad \text { if same parity } \\
\mathfrak{I}_{\max }^{m_{1}+m_{2}} \backslash\left\{\left[\mathrm{Z}_{m_{1}+m_{2}}\right]\right\}, \quad \text { if different parity }\end{array}\right.$ \\
\hline
\end{tabular}

with the two degenerate cases

$$
\begin{aligned}
& \mathfrak{I}\left(S^{2}\left(\mathbb{S}^{1}\right)\right)=\left\{\left[\mathrm{D}_{2}\right],[\mathrm{O}(2)]\right\} \\
& \mathfrak{I}\left(S^{2}\left(\mathbb{S}^{2}\right)\right)=\left\{\left[\mathrm{Z}_{2}\right],\left[\mathrm{D}_{2}\right],\left[\mathrm{D}_{4}\right],[\mathrm{O}(2)]\right\} .
\end{aligned}
$$

The following lemma can be obtained by induction on $p$.

Lemma 2.5. For each integer $p \geq 1$, we have

$$
\begin{aligned}
\mathfrak{I}\left(\mathbb{S}^{2 p}\right) & =\left\{\left[\mathrm{Z}_{2 k}\right],\left[\mathrm{D}_{2 k}\right]\right\}_{1 \leq k \leq\left\lfloor\frac{p}{2}\right\rfloor} \cup\left\{\left[\mathrm{D}_{2 k}\right]\right\}_{\left\lfloor\frac{p}{2}\right\rfloor+1 \leq k \leq p} \cup\{[\mathrm{O}(2)]\} \\
\mathfrak{I}\left(\mathbb{S}^{2 p+1}\right) & =\left\{[1],\left[\mathrm{Z}_{2}^{\sigma_{x}}\right],[\mathrm{O}(2)]\right\} \cup\left\{\left[\mathrm{Z}_{\left.\left.\mathcal{O}\left(\left\lfloor\frac{2 k+1}{3}\right\rfloor\right)\right],\left[\mathrm{D}_{2 k+1}\right]\right\}_{1 \leq k \leq p}}\right.\right.
\end{aligned}
$$

where $\lfloor x\rfloor$ is the integer part of $x$ and the function $\mathcal{O}($.$) is defined as$

$$
\mathcal{O}(k):=\left\{\begin{array}{ll}
k, & \text { if } k \text { is odd } \\
0, & \text { if } k \text { is even }
\end{array} .\right.
$$

Let us now consider some explicit examples.

\subsection{Examples}

Classical elasticity tensors The most famous example in the literature is the space of elasticity tensors $[10,23,37,14,19]$, namely

$$
\mathbb{E l a}:=S^{2}\left(\mathbb{S}^{2}\right)=\left\{\mathrm{E}_{i j k l} \in \otimes^{4}\left(\mathbb{R}^{2}\right) \mid \mathrm{E}_{\underline{(i j)}} \underline{(k l)}\right\} .
$$

The symmetry classes of $\mathbb{E l a}$ are summed-up in the following table ${ }^{2}$ :

\section{Table 3}

\begin{tabular}{|c||c|c|c|c|}
\hline Name & Monoclinic, Diagonal & Orthotropic & Tetragonal & Isotropic \\
\hline$\left[\mathrm{G}_{\mathrm{E}}\right]$ & {$\left[\mathrm{Z}_{2}\right]$} & {$\left[\mathrm{D}_{2}\right]$} & {$\left[\mathrm{D}_{4}\right]$} & {$[\mathrm{O}(2)]$} \\
\hline$\#_{\text {indep }}(\mathrm{E})$ & $6(5)$ & 4 & 3 & 2 \\
\hline
\end{tabular}

The in-parenthesis number indicates the minimal number of independent components of the matrix in an adapted basis. Some observations should be made here:

- there is no odd-order rotational invariant symmetry class;

- each elasticity tensor is at least in $\left[\mathrm{Z}_{2}\right]$;

- there is no tetrachiral class $\left[\mathrm{Z}_{4}\right]$.

Higher order elasticity tensors Other examples involve higher order tensors and are borrowed from Mindlin strain gradient elasticity [27, 28]. Some works on its bi-dimensional anisotropic formulation have recently been conducted in $[3,4]$. In this model, aside from the classical fourth-order elasticity tensor, a fifth- and a sixth-order tensor are added. Those tensors belong to:

- the space of coupling elasticity tensors:

$$
\text { Cou }=\left\{\mathrm{M}_{i j k l m} \in \otimes^{5}\left(\mathbb{R}^{2}\right) \mid \mathrm{M}_{(i j)(k l) m}\right\} ;
$$


- the space of second-order elasticity tensors:

$$
\operatorname{Str}=\left\{\mathrm{A}_{i j k l m n} \in \otimes^{6}\left(\mathbb{R}^{2}\right) \mid \mathrm{A}_{\underline{(i j) k} \underline{\underline{(l m) n}}}\right\} .
$$

Hence the first space is the coupling space $\mathbb{S}^{2} \otimes\left(\mathbb{S}^{2} \otimes \mathbb{K}^{1}\right)$, while the second space is $S^{2}\left(\mathbb{S}^{2} \otimes \mathbb{K}^{1}\right)$. For the fifth-order tensor space $\mathbb{C}$ ou, we get:

Table 4

\begin{tabular}{|c||c|c|c|c|c|c|}
\hline Name & Biclinic & Rectangular & Trichiral & Trigonal & Pentagonal & Isotropic \\
\hline$\left[\mathrm{G}_{\mathrm{M}}\right]$ & {$[1]$} & {$\left[\mathrm{Z}_{2}^{\sigma_{x}}\right]$} & {$\left[\mathrm{Z}_{3}\right]$} & {$\left[\mathrm{D}_{3}\right]$} & {$\left[\mathrm{D}_{5}\right]$} & {$[\mathrm{O}(2)]$} \\
\hline$\#_{\text {indep }}(\mathrm{M})$ & $18(17)$ & 9 & $6(5)$ & 3 & 1 & 0 \\
\hline
\end{tabular}

In the previous table, some observations can be made:

- there is no even-order invariant rotational symmetry class;

- there is no pentachiral class $\left[\mathrm{Z}_{5}\right]$.

Now for $\mathbb{S t r}$, we have:

Table 5

\begin{tabular}{|c||c|c|c|c|}
\hline Name & Monoclinic, Diagonal & Orthotropic & Tetrachiral & Tetragonal \\
\hline$\left[\mathrm{G}_{\mathrm{A}}\right]$ & {$\left[\mathrm{Z}_{2}\right]$} & {$\left[\mathrm{D}_{2}\right]$} & {$\left[\mathrm{Z}_{4}\right]$} & {$\left[\mathrm{D}_{4}\right]$} \\
\hline$\#_{\text {indep }}(\mathrm{A})$ & $21(20)$ & 12 & $9(8)$ & 6 \\
\hline \hline Name & Hexachiral & Hexagonal & Hemitropic & Isotropic \\
\hline$\left[\mathrm{G}_{\mathrm{A}}\right]$ & {$\left[\mathrm{Z}_{6}\right]$} & {$\left[\mathrm{D}_{6}\right]$} & {$[\mathrm{SO}(2)]$} & {$[\mathrm{O}(2)]$} \\
\hline$\#_{\text {indep }}(\mathrm{A})$ & $7(6)$ & 5 & 5 & 4 \\
\hline
\end{tabular}

and we notice that:

- there is no odd-order rotational invariant symmetry class;

- each Str-tensor is at least in $\left[\mathrm{Z}_{2}\right]$;

- a "surprising" hemitropic class $[\mathrm{SO}(2)]$ appears.

The proofs of these results, as well as the calculation of the number of independent components, for each symmetry class rely on the harmonic decomposition of tensor spaces, which is introduced in the next section. An algorithm to compute this decomposition will be provided, together with a formula which gives the dimension of the fixed point spaces (elements that are fixed by the action of a given subgroup $H \subset \mathrm{O}(2)$ ). From a mechanical point of view, this dimension is the number of physical parameters required to describe a tensor in each symmetry class. This information was provided in the last line of the former tables.

\section{Harmonic decomposition}

In order to properly set up the problem, tensor spaces should be decomposed into a collection of elementary spaces called irreducible representations of $\mathrm{O}(2)$. We refer the reader to subsection A.2 for an overview of the general mathematical framework, and focus here on the particular case of $\mathrm{O}(2)$.

Example 3.1. Let us illustrate this decomposition in the special case of second-order symmetric tensors. Any element $\mathrm{T} \in S^{2}\left(\mathbb{R}^{2}\right)$ admits the following decomposition

$$
\mathrm{T}=\mathrm{K}^{2}+\frac{1}{2} \mathrm{~K}^{0} \mathrm{q}=\phi\left(\mathrm{K}^{2}, \mathrm{~K}^{0}\right)
$$

where $\mathrm{K}^{2} \in \mathbb{K}^{2}$ and $\mathrm{K}^{0} \in \mathbb{K}^{0}$ are, respectively, the $2 \mathrm{D}$ deviatoric and $1 \mathrm{D}$ spheric part of $\mathrm{T}$ and $\mathrm{q}$ is the identity tensor. They are defined by the following formulas:

$$
\mathrm{K}^{0}:=\mathrm{T}_{i i} \quad ; \quad \mathrm{K}_{i j}^{2}:=\mathrm{T}_{i j}-\frac{1}{2} \mathrm{~K}^{0} \delta_{i j} .
$$

Prepared using sagej.cls 
The linear mapping $\phi$, defined by expression (3.1), is an isomorphism between the direct sum $\mathbb{K}^{2} \oplus \mathbb{K}^{0}$ and $S^{2}\left(\mathbb{R}^{2}\right)$

$$
\mathbb{K}^{2} \oplus \mathbb{K}^{0} \simeq S^{2}\left(\mathbb{R}^{2}\right)
$$

This decomposition is moreover $\mathrm{O}(2)$-equivariant, or expressed in other way: the components $\left(\mathrm{K}^{0}, \mathrm{~K}^{2}\right)$ are covariant with $\mathrm{T}$ under the $\mathrm{O}(2)$-action, i.e.

$$
\mathrm{QTQ}^{T}=\phi\left(\mathrm{QK}^{2} \mathrm{Q}^{T}, \mathrm{~K}^{0}\right), \quad \forall \mathrm{Q} \in \mathrm{O}(2), \forall \mathrm{T} \in S^{2}\left(\mathbb{R}^{2}\right) .
$$

This decomposition is also irreducible, which means that those tensors cannot be split into smaller tensors satisfying this property. Let us now extend this procedure to higher-order tensors.

The first step to achieve is to generalize the notion of deviator.

\section{1 $\mathrm{O}(2)$-irreducible representations}

For each integer $n \geq 1$, there is a natural $\mathrm{O}(2)$-representation $\rho_{n}$ on the two dimensional space $\mathbb{R}^{2}$ given by

$$
\rho_{n}\left(r_{\theta}\right):=\left(\begin{array}{cc}
\cos n \theta & -\sin n \theta \\
\sin n \theta & \cos n \theta
\end{array}\right), \quad \rho_{n}\left(\sigma_{x}\right):=\left(\begin{array}{cc}
1 & 0 \\
0 & -1
\end{array}\right) .
$$

There are also two natural $\mathrm{O}(2)$-representations on the one dimensional space $\mathbb{R}$ : one of them $\rho_{0}$ is the trivial representation, and the other one $\rho_{-1}$ is the multiplication by $\operatorname{det}(\mathrm{Q})$. All these representations are irreducible and each irreducible representation of $\mathrm{O}(2)$ is equivalent to one of them.

Given $n \geq 1$, let $\mathcal{H}^{n}$ be the space of homogeneous polynomials of degree $n$ in two variables. There is a natural action $\tilde{\rho}_{n}$ of the orthogonal group $\mathrm{O}(2)$ on $\mathcal{H}^{n}$ :

$$
\left(\tilde{\rho}_{n}(\mathrm{Q})(\mathrm{P})\right)(\mathbf{x}):=\mathrm{P}\left(\rho_{n}(\mathrm{Q})^{-1}(\mathbf{x})\right), \quad \mathrm{P} \in \mathcal{H}^{n}, \quad \mathbf{x} \in \mathbb{R}^{2}, \quad \mathrm{Q} \in \mathrm{O}(2) .
$$

The subspace $\mathcal{K}^{n} \subset \mathcal{H}^{n}$ of harmonic polynomials (with vanishing Laplacian) is invariant under this action. Since any (real) harmonic function on $\mathbb{R}^{2}$ is the real or imaginary part of a holomorphic function, we conclude that $\mathcal{K}^{n}$ is generated by $\operatorname{Re}\left(z^{n}\right)$ and $\operatorname{Im}\left(z^{n}\right)$. In this basis, the action of $\mathrm{O}(2)$ is precisely $\left(\mathbb{R}^{2}, \rho_{n}\right)$ introduced in $(3.2)$.

A third model for this irreducible representation is given by $\mathbb{K}^{n}$, the space of harmonic tensors; i.e $n$-th order totally symmetric and traceless tensors on $\mathbb{R}^{2}$ (with its natural $\mathrm{O}(2)$-action), where the trace of an $n$-th order totally symmetric tensor $\mathrm{T}$ is defined as the $(n-2)$-th order tensor:

$$
(\operatorname{tr} \mathrm{T})\left(\mathbf{x}_{1}, \ldots, \mathbf{x}_{n-2}\right):=\sum_{i=1}^{2} \mathrm{~T}\left(\mathbf{x}_{1}, \ldots, \mathbf{x}_{n-2}, \mathbf{e}_{i}, \mathbf{e}_{i}\right) .
$$

Remark 3.2. The link between the space of harmonic tensors $\mathbb{K}^{n}$ and the space of homogeneous harmonic polynomials $\mathcal{K}^{n}$ is described as follows. First, note that to each totally symmetric tensor $\mathrm{T}$ corresponds a homogeneous polynomials $\mathrm{P}$, given by $\mathrm{P}(\mathbf{x}):=\mathrm{T}(\mathbf{x}, \ldots, \mathbf{x})$. This correspondence can be shown to induce an $\mathrm{O}(2)$-equivariant isomorphism $\Phi$ between the spaces $\mathbb{S}^{n}$ and $\mathcal{H}^{n}$ and its inverse can be obtained explicitly by polarization $[31,6]$. When restricted to the subspace of traceless tensors, the image of $\Phi$ corresponds to the space of harmonic polynomials, since

$$
(\triangle \mathrm{P})(\mathbf{x})=n(n-1)(\operatorname{tr} \mathrm{T})(\mathbf{x}, \ldots, \mathbf{x}) .
$$

Finally, we set $\mathbb{K}^{0}=\mathbb{R}$ with the identity $\mathrm{O}(2)$-action and $\mathbb{K}^{-1}=\mathbb{R}$ with the determinant $\mathrm{O}(2)$ action. We can interpret these representations as follows:

$\mathbb{K}^{-1}$ is the space of pseudo-scalars, or hemitropic coefficients;

$\mathbb{K}^{0}$ is the space of scalars, that is isotropic coefficients;

$\mathbb{K}^{1}$ is the space of vectors;

$\mathbb{K}^{2}$ is the space of deviators;

$\mathbb{K}^{n}(n \geq 3)$ is the space of $n$-th order deviators. ${ }^{3}$ 


\subsection{Decomposition into irreducible representations}

We can decompose any representation $\mathbb{T}^{n} \subset \mathbb{G}^{n}$ of the group $\mathrm{O}(2)$ or $\mathrm{SO}(2)$ into irreducible pieces, that is into harmonic tensor spaces $\mathbb{K}^{k}[17]$. We shall write

$$
\mathbb{T}^{n} \simeq \bigoplus_{i=1}^{q} \mathbb{K}^{k_{i}}
$$

Since several pieces may be isomorphic to the same harmonic tensor space $\mathbb{K}^{k}$, it is preferable to group all these pieces into one space

$$
\alpha_{k} \mathbb{K}^{k}:=\bigoplus_{l=1}^{\alpha_{k}} \mathbb{K}^{k}
$$

called an isotypic component (of type $\mathbb{K}^{k}$ ) of the representation $\mathbb{T}^{n}$. The integer $\alpha_{k}$ indicates the multiplicity of $\mathbb{K}^{k}$ into the decomposition (3.3) (it is zero if the representation $\mathbb{K}^{k}$ does not appear in (3.3)). We shall write therefore

$$
\mathbb{T}^{n} \simeq \bigoplus_{k} \alpha_{k} \mathbb{K}^{k}
$$

where it is understood that only a finite number of $\alpha_{k}$ do not vanish.

Remark 3.3. It is worth noting that the decomposition (3.3) is uniquely defined only if each $k_{i}$ appears once (c.f. [22]). At the opposite the global structure of the decomposition (3.4) is uniquely defined.

To determine the symmetry classes of a tensor space, the computation of an explicit isomorphism that realizes the harmonic decomposition (3.3) is not mandatory, only the knowledge of the sequence $\left(\alpha_{k}\right)$ in the isotypic decomposition (3.4) is required. In the next section, an easy way to determine this sequence is presented.

\subsection{Clebsch-Gordan formula}

The structure of the isotypic decomposition can easily be determined using the Clesbch-Gordan formula, which is a direct consequence of the character formula for $\mathrm{O}(2)$ representations, as detailed in the Appendix A.

Lemma 3.4. For every integers $m>0$ and $n>0$, we have the following isotypic decompositions

\begin{tabular}{|c|c|c|c|}
\hline$\otimes$ & $\mathbb{K}^{n}$ & $\mathbb{K}^{0}$ & $\mathbb{K}^{-1}$ \\
\hline $\mathbb{K}^{m}$ & $\left\{\begin{array}{l}\mathbb{K}^{2 n} \oplus \mathbb{K}^{0} \oplus \mathbb{K}^{-1}, n=m ; \\
\mathbb{K}^{n+m} \oplus \mathbb{K}^{|n-m|}, n \neq m ;\end{array}\right.$ & $\mathbb{K}^{m}$ & $\mathbb{K}^{m}$ \\
\hline $\mathbb{K}^{0}$ & $\mathbb{K}^{n}$ & $\mathbb{K}^{0}$ & $\mathbb{K}^{-1}$ \\
\hline $\mathbb{K}^{-1}$ & $\mathbb{K}^{n}$ & $\mathbb{K}^{-1}$ & $\mathbb{K}^{0}$ \\
\hline
\end{tabular}

\section{Table 6}

Note that the tensor product between two identical spaces can be decomposed into a symmetric $\left(S^{2}\right)$ and an antisymmetric product $\left(\Lambda^{2}\right)$ :

$$
\forall n \geq 1, \quad \mathbb{K}^{n} \otimes \mathbb{K}^{n}=S^{2}\left(\mathbb{K}^{n}\right) \oplus \Lambda^{2}\left(\mathbb{K}^{n}\right) .
$$

Therefore Table 6 can be completed by the following ones:

Lemma 3.5. For every integer $n \geq 1$, we have the following isotypic decompositions, in which meaningless products are indicated by $\times$

\begin{tabular}{|c|c|c|c|}
\hline$S^{2}$ & $\mathbb{K}^{n}$ & $\mathbb{K}^{0}$ & $\mathbb{K}^{-1}$ \\
\hline $\mathbb{K}^{n}$ & $\mathbb{K}^{2 n} \oplus \mathbb{K}^{0}$ & $\times$ & $\times$ \\
\hline $\mathbb{K}^{0}$ & $\times$ & $\mathbb{K}^{0}$ & $\times$ \\
\hline $\mathbb{K}^{-1}$ & $\times$ & $\times$ & $\mathbb{K}^{0}$ \\
\hline
\end{tabular}

\begin{tabular}{|c|c|c|c|}
\hline$\Lambda^{2}$ & $\mathbb{K}^{n}$ & $\mathbb{K}^{0}$ & $\mathbb{K}^{-1}$ \\
\hline $\mathbb{K}^{n}$ & $\mathbb{K}^{-1}$ & $\times$ & $\times$ \\
\hline $\mathbb{K}^{0}$ & $\times$ & 0 & $\times$ \\
\hline $\mathbb{K}^{-1}$ & $\times$ & $\times$ & 0 \\
\hline
\end{tabular}

Prepared using sagej.cls 
Example 3.6. Consider the space of symmetric second-order tensors $S^{2}\left(\mathbb{R}^{2}\right)=S^{2}\left(\mathbb{K}^{1}\right)$. From Lemma 3.5, we get:

$$
S^{2}\left(\mathbb{K}^{1}\right) \simeq \mathbb{K}^{2} \oplus \mathbb{K}^{0}
$$

The classical decomposition between a deviatoric part and a spherical one is retrieved. For a more complex example, we consider $\mathbb{E} l a=\mathbb{T}_{\underline{(i j)}} \underline{(k l)}$. This space can be viewed as $S^{2}\left(S^{2}\left(\mathbb{R}^{2}\right)\right)[1]$ :

$$
\begin{aligned}
\mathbb{E} \mathrm{la} \simeq S^{2}\left(S^{2}\left(\mathbb{R}^{2}\right)\right) & \simeq S^{2}\left(\mathbb{K}^{2} \oplus \mathbb{K}^{0}\right) \simeq S^{2}\left(\mathbb{K}^{2}\right) \oplus S^{2}\left(\mathbb{K}^{0}\right) \oplus\left(\mathbb{K}^{2} \otimes \mathbb{K}^{0}\right) \\
& \simeq \mathbb{K}^{4} \oplus \mathbb{K}^{0} \oplus \mathbb{K}^{0} \oplus \mathbb{K}^{2} \simeq \mathbb{K}^{4} \oplus \mathbb{K}^{2} \oplus 2 \mathbb{K}^{0}
\end{aligned}
$$

Hence we recover the structure of the well-known harmonic decomposition of plane elasticity [10, $37,14,19]$ without any computation.

Other harmonic structures can be found in the following table:

\begin{tabular}{|l|l|l|l|}
\hline Symmetries & Tensor space & Isotypic dec. & Physical meaning \\
\hline $\mathbb{T}_{i}$ & $\mathbb{R}^{2}$ & $\mathbb{K}^{1}$ & Displacement \\
\hline $\mathbb{T}_{(i j)}$ & $S^{2}\left(\mathbb{R}^{2}\right)$ & $\mathbb{K}^{2} \oplus \mathbb{K}^{0}$ & Conduction \\
\hline $\mathbb{T}_{(i j) k}$ & $S^{2}\left(\mathbb{R}^{2}\right) \otimes \mathbb{R}^{2}$ & $\mathbb{K}^{3} \oplus 2 \mathbb{K}^{1}$ & Piezoelectricity [36] \\
\hline $\mathbb{T}_{(i j)} \underline{(l m)}$ & $S^{2}\left(S^{2}\left(\mathbb{R}^{2}\right)\right)$ & $\mathbb{K}^{4} \oplus \mathbb{K}^{2} \oplus 2 \mathbb{K}^{0}$ & Elasticity [10, 37, 14, 19] \\
\hline $\mathbb{T}_{(i j)(k l) m}$ & $S^{2}\left(S^{2}\left(\mathbb{R}^{2}\right)\right) \otimes \mathbb{R}^{2}$ & $\mathbb{K}^{5} \oplus 3 \mathbb{K}^{3} \oplus 5 \mathbb{K}^{1}$ & Strain-gradient coupling [4] \\
\hline $\mathbb{T}_{\overline{(i j)} \underline{(k l) m}}$ & $\Lambda^{2}\left(S^{2}\left(\mathbb{R}^{2}\right)\right) \otimes \mathbb{R}^{2}$ & $\mathbb{K}^{3} \oplus 2 \mathbb{K}^{1}$ & Gyrotropicity [4] \\
\hline $\mathbb{T}_{\underline{(i j) k} \underline{\underline{(l m) n}}}$ & $S^{2}\left(S^{2}\left(\mathbb{R}^{2}\right) \otimes \mathbb{R}^{2}\right)$ & $\begin{array}{l}\mathbb{K}^{6} \oplus 2 \mathbb{K}^{4} \oplus 5 \mathbb{K}^{2} \oplus \\
4 \mathbb{K}^{0} \oplus \mathbb{K}^{-1}\end{array}$ & Strain-gradient elasticity [3] \\
\hline
\end{tabular}

Table 7

As can be observed in previous examples, the decomposition of even-order tensor spaces contains only even-order harmonic spaces, and the same occurs for odd-order tensors. This fact is indeed a general fact and can be proven using the Clebch-Gordan decomposition (c.f. Lemma 3.9).

\subsection{Isotypic decomposition of usual tensor spaces}

Following observations made in subsection 2.3 to demonstrate our main theorem, the harmonic decomposition of $\mathbb{S}^{n}, S^{2}\left(\mathbb{S}^{n}\right)$ and $\mathbb{G}^{n}$ have to be determined, which will be achieved in this section.

Lemma 3.7. Let $\mathbb{S}^{n}=S^{n}\left(\mathbb{R}^{2}\right)$ be the space of $n$-th order completely symmetric tensors. Then:

$$
\mathbb{S}^{2 q} \simeq \bigoplus_{k=0}^{q} \mathbb{K}^{2 k}, \quad \mathbb{S}^{2 q+1} \simeq \bigoplus_{k=0}^{q} \mathbb{K}^{2 k+1}
$$

Proof. It is a classical result (see [33] for instance) that any homogeneous polynomial $\mathrm{F}$ of degree $n$ can be decomposed uniquely as

$$
\mathrm{F}=\mathrm{P}_{0}+\mathbf{q P}_{1}+\cdots+\mathbf{q}^{r} \mathrm{P}_{r}
$$

where $\mathbf{q}(x, y)=x^{2}+y^{2}, \mathrm{P}_{k}$ is an harmonic polynomial of degree $n-2 k$ and $r=[n / 2]$. The conclusion follows using the isomorphism between the space of totally symmetric tensors $\mathbb{S}^{n}$ and the space $\mathcal{H}^{n}$ of homogeneous polynomials of degree $n$, described in remark 3.2. 
Lemma 3.8. The isotypic decompositions of $S^{2}\left(\mathbb{S}^{2 p}\right)(p \geq 1)$ and $S^{2}\left(\mathbb{S}^{2 q+1}\right)(q \geq 0)$ are given by:

$$
\begin{aligned}
S^{2}\left(\mathbb{S}^{2 p}\right) & \simeq(p+1) \mathbb{K}^{0} \oplus\left(\bigoplus_{k=1}^{p}(p-k+1)\left(\mathbb{K}^{4 k} \oplus \mathbb{K}^{4 k-2}\right)\right), \\
S^{2}\left(\mathbb{S}^{2 q+1}\right) & \simeq \bigoplus_{k=0}^{q}(q-k+1)\left(\mathbb{K}^{4 k+2} \oplus \mathbb{K}^{4 k}\right) .
\end{aligned}
$$

Proof. It suffices to note that

$$
S^{2}\left(\mathbb{S}^{2 p}\right)=\bigoplus_{k=0}^{p} \mathbb{S}^{4 k}
$$

and to apply Lemma 3.7.

Lemma 3.9. For any integer $n \geq 1$, the isotypic decomposition of $\mathbb{G}^{n}=\otimes^{n} \mathbb{R}^{2}$ is given by:

$$
\mathbb{G}^{n} \simeq\left\{\begin{array}{l}
\left(\begin{array}{c}
2 p-1 \\
p
\end{array}\right)\left(\mathbb{K}^{-1} \oplus \mathbb{K}^{0}\right) \oplus\left(\bigoplus_{k=1}^{p}\left(\begin{array}{c}
2 p \\
p+k
\end{array}\right) \mathbb{K}^{2 k}\right), \quad \text { if } n=2 p, p>0 \\
\bigoplus_{k=0}^{q}\left(\begin{array}{c}
2 q+1 \\
q+1+k
\end{array}\right) \mathbb{K}^{2 k+1}, \quad \text { if } n=2 q+1, q \geq 0
\end{array}\right.
$$

where $\left(\begin{array}{l}k \\ n\end{array}\right)$ is the binomial coefficient.

Proof. It is an application of the character formula given in subsection A.3, using the development of the character $\chi_{\mathbb{G}^{n}}$ for the $\mathrm{O}(2)$-representation $\mathbb{G}^{n}=\otimes^{n} \mathbb{R}^{2}$

$$
\chi_{\mathbb{G}^{n}}\left(r_{\theta}\right)=(2 \cos \theta)^{n}=\left(e^{i \theta}+e^{-i \theta}\right)^{n}, \quad \chi_{\mathbb{G}^{n}}\left(\sigma_{x} r_{\theta}\right)=0 .
$$

We end this section with some results about dimensions of fixed point spaces. As it will be illustrated below, this knowledge is not sufficient to determine the symmetry classes. Nevertheless, once the symmetry classes is determined, these formula are needed to establish Table 3 , Table 4 and Table 5 .

\subsection{Fixed-point spaces}

We introduce here formulas which allow to easily determine the number of parameters of a given $H$-invariant tensor. Recall that given a tensorial representation $\mathbb{T}^{n}$ of $n$-th order tensors and a subgroup $H \subset \mathrm{O}(2)$, the fixed-point space $\left(\mathbb{T}^{n}\right)^{H}$ associated to $H$ is defined as

$$
\left(\mathbb{T}^{n}\right)^{H}:=\left\{\mathrm{T} \in \mathbb{T}^{n}, \quad \mathrm{Q} \cdot \mathrm{T}=\mathrm{T}, \quad \forall \mathrm{Q} \in H\right\} .
$$

The dimension of $\left(\mathbb{T}^{n}\right)^{H}$ can easily be computed.

Lemma 3.10. Let

$$
\mathbb{T}^{n} \simeq \bigoplus_{k} \alpha_{k} \mathbb{K}^{k}
$$

where $\alpha_{k}$ is the multiplicity of $\mathbb{K}^{k}$ in the decomposition. Then

$$
\operatorname{dim}\left(\mathbb{T}^{n}\right)^{Z_{p}}=\alpha_{-1}+\alpha_{0}+2 \sum_{\substack{k \geq 1 \\ p \mid k}} \alpha_{k} \quad ; \quad \operatorname{dim}\left(\mathbb{T}^{n}\right)^{\mathrm{D}_{p}}=\alpha_{0}+\sum_{\substack{k \geq 1 \\ p \mid k}} \alpha_{k}
$$

where the notation $p \mid k$ means $p$ divides $k$ and with the obvious limit cases:

$$
\operatorname{dim}\left(\mathbb{T}^{n}\right)^{\mathrm{SO}(2)}=\alpha_{-1}+\alpha_{0} \quad ; \quad \operatorname{dim}\left(\mathbb{T}^{n}\right)^{\mathrm{O}(2)}=\alpha_{0} .
$$

Prepared using sagej.cls 
Remark 3.11. It is important to note that the knowledge of the different fixed-point spaces dimensions does not allow to conclude on the number and type of symmetry classes. For example, by using these formulas we establish that:

- The dimension of $(\mathbb{S t r})^{\mathrm{D}_{6}}$ and $(\mathbb{S t r})^{\mathrm{SO}(2)}$ are identical, but the symmetry classes $\left[\mathrm{D}_{6}\right]$ and $[\mathrm{SO}(2)]$ are distinct;

- The dimension of $(\mathbb{E} l a)^{\mathrm{Z}_{4}}$ and $(\mathbb{E} l a)^{\mathrm{D}_{4}}$ are different, but both elements belongs to the same class $\left[\mathrm{D}_{4}\right]$.

In the next section, a rigorous method to determine the symmetry classes of a tensor representation will be presented.

\section{Symmetry classes and clips operation}

The purpose of this section is to present some general results about symmetry classes of constitutive tensor spaces under $O(2)$-group action . In subsection 4.1, we describe the symmetry classes irreducible $\mathrm{O}(2)$-representations. In subsection 4.2, we introduce the clips operator, a tool to compute the symmetry classes of the direct sum of two representations. Finally, in subsection 4.3, we present an algorithm to compute the symmetry classes of every reducible $\mathrm{O}(2)$-representation. The proof of Theorem 2.4 and subsequent results for tensor spaces are provided in subsection 4.4.

\subsection{Symmetry classes of irreducible $\mathrm{O}(2)$-representation}

Before computing isotropy classes of a reducible $\mathrm{O}(2)$-representation, we list the isotropy classes of all the irreducible $\mathrm{O}(2)$-representations. These classes are given by the following theorem.

Theorem 4.1. The symmetry classes of the $\mathrm{O}(2)$-irreducible space $\mathbb{K}^{n}$ are:

$$
\mathfrak{I}\left(\mathbb{K}^{n}\right)=\left\{\begin{array}{l}
\{[\mathrm{SO}(2)],[\mathrm{O}(2)]\} ; \quad \text { if } n=-1 \\
\{[\mathrm{O}(2)]\} ; \quad \text { if } n=0 \\
\left\{\left[\mathrm{D}_{n}\right],[\mathrm{O}(2)]\right\} ; \quad \text { if } n \geq 1
\end{array}\right.
$$

with the convention $D_{1}=\mathrm{Z}_{2}^{\sigma_{x}}$.

Proof. For $n \geq 1$, there are two possibilities. If the vector $\mathbf{v} \in \mathbb{R}^{2}$ is null its symmetry class is $[\mathrm{O}(2)]$. In the other case, there is an element $\mathrm{Q} \in \mathrm{O}(2) \operatorname{such}$ that $\mathrm{Q} \cdot \mathbf{v}=(r, 0)$ (with $r>0$ ), hence $\mathbf{v}$ is $Z_{2}^{\sigma_{x}}$-invariant. Furthermore, since $\mathbf{v}$ belongs to $\mathbb{K}^{n}$, a rotation of angle $\theta$ turns $\mathbf{v}$ by an angle $n \theta$. Hence, $\mathbf{v}$ is invariant by the rotation of angle $\frac{2 \pi}{n}$, i.e. $\mathbf{v}$ is $Z_{n}$-invariant. Thus, $\mathbf{v}$ belongs to the symmetry class $\left[D_{n}\right]$. The other cases are obvious.

\subsection{Clips operator}

Every $\mathrm{O}(2)$-representation $\mathbb{V}$ can be decomposed into a direct sum of irreducible $\mathrm{O}(2)$ representations $\mathbb{V}_{i}(i=1, \ldots, n)$. To obtain the symmetry class of $\mathbb{V}$, we need therefore to compute the symmetry classes of a direct sum $\mathbb{V}_{1} \oplus \mathbb{V}_{2}$, knowing independently the symmetry classes of each space $\mathbb{V}_{i}$. This can be achieved using the clips operator (which was first introduced in [29,30] for $\mathrm{SO}(3)$ and $\mathrm{O}(3)$ representations).

Lemma 4.2. Let $\mathbb{V}$ be a representation of a group $G$ that split into a direct sum of two $G$-invariant subspaces

$$
\mathbb{V}=\mathbb{V}_{1} \oplus \mathbb{V}_{2} \text { where } g \cdot \mathbb{V}_{1} \subset \mathbb{V}_{1} \text { and } g \cdot \mathbb{V}_{2} \subset \mathbb{V}_{2}, \quad \forall g \in G \text {. }
$$

If $\mathfrak{I}$ denotes the set of all isotropy classes associated to $\mathbb{V}, \mathfrak{I}_{i}$ the set of all isotropy classes associated to $\mathbb{V}_{i}(i=1,2)$, then $[H] \in \mathfrak{I}$ if and only if there exist $\left[H_{1}\right] \in \mathfrak{I}_{1}$ and $\left[H_{2}\right] \in \mathfrak{I}_{2}$ such that $H=H_{1} \cap H_{2}$. 
Lemma 4.2 shows that the isotropy classes of a direct sum are related to intersections of isotropy subgroups. But as intersection of classes is meaningless, the result cannot be directly extended. The clips operator solves this problem.

Definition 4.3. Clips Operator. For every conjugacy classes $\left[H_{1}\right]$ and $\left[H_{2}\right]$, we define the clips operator of $\left[H_{1}\right]$ and $\left[H_{2}\right]$, noted $\left[H_{1}\right] \odot\left[H_{2}\right]$, to be

$$
\left[H_{1}\right] \odot\left[H_{2}\right]:=\left\{\left[H_{1} \cap g H_{2} g^{-1}\right] \text { for all } g \in G\right\}
$$

which is a set of conjugacy classes.

One can observe some immediate properties:

Proposition 4.4. The clips operation is commutative.

Proposition 4.5. For every conjugacy class $[H]$ of a group $G$, we have

$$
[1] \odot[H]=\{[1]\} \text { and }[G] \odot[H]=\{[H]\} \text {. }
$$

We also have the following result which is a corollary of Lemma 4.2.

Corollary 4.6. Given two G-representations $\mathbb{V}_{1}$ and $\mathbb{V}_{2}$, if $\mathfrak{I}_{k}$ denotes the set of isotropy classes of $\mathbb{V}_{k}$ for $k=1,2$, then

$$
\mathfrak{I}_{1} \odot \mathfrak{I}_{2}:=\bigcup_{\left[H_{1}\right] \in \mathfrak{I}_{1},\left[H_{2}\right] \in \mathfrak{I}_{2}}\left[H_{1}\right] \odot\left[H_{2}\right]
$$

is the set of all the isotropy classes of $\mathbb{V}_{1} \oplus \mathbb{V}_{2}$.

\subsection{Symmetry classes of a reducible $\mathrm{O}(2)$-representation}

The symmetry classes of a reducible $\mathrm{O}(2)$-representation can now be computed using iterated clips operations and the following table:

Theorem 4.7. Clips operations between $\mathrm{O}(2)$-closed subgroups are given in the following table:

\begin{tabular}{|c|c|c|c|c|c|c|}
\hline$\odot$ & {$[1]$} & {$\left[\mathrm{Z}_{2}^{\sigma_{x}}\right]$} & {$\left[\mathrm{Z}_{n}\right]$} & {$\left[\mathrm{D}_{n}\right]$} & {$[\mathrm{SO}(2)]$} & {$[\mathrm{O}(2)]$} \\
\hline$[1]$ & {$[1]$} & & & & & \\
\hline$\left[\mathrm{Z}_{2}^{\sigma_{x}}\right]$ & {$[1]$} & {$[1],\left[\mathrm{Z}_{2}^{\sigma_{x}}\right]$} & & & & \\
\hline$\left[\mathrm{Z}_{m}\right]$ & {$[1]$} & {$[1]$} & {$\left[\mathrm{Z}_{d(n, m)}\right]$} & & & \\
\hline$\left[\mathrm{D}_{m}\right]$ & {$[1]$} & {$[1],\left[\mathrm{Z}_{2}^{\sigma_{x}}\right]$} & {$\left[\mathrm{Z}_{d(n, m)}\right]$} & {$\left[\mathrm{Z}_{d(n, m)}\right],\left[\mathrm{D}_{d(n, m)}\right]$} & & \\
\hline$[\mathrm{SO}(2)]$ & {$[1]$} & {$[1]$} & {$\left[\mathrm{Z}_{n}\right]$} & {$\left[\mathrm{Z}_{n}\right]$} & {$[\mathrm{SO}(2)]$} & \\
\hline$[\mathrm{O}(2)]$ & {$[1]$} & {$\left[\mathrm{Z}_{2}^{\sigma_{x}}\right]$} & {$\left[\mathrm{Z}_{n}\right]$} & {$\left[\mathrm{D}_{n}\right]$} & {$[\mathrm{SO}(2)]$} & {$[\mathrm{O}(2)]$} \\
\hline
\end{tabular}

Table 8. Clips operation on $\mathrm{O}(2)$ closed subgroups

where $\mathrm{Z}_{1}:=1, \mathrm{D}_{1}:=\mathrm{Z}_{2}^{\sigma_{x}}$ and $d(n, m):=\operatorname{gcd}(n, m)$.

Due to the commutativity of the clips operator the table is symmetric and, accordingly, only its lower half part has been filled.

Proof. First we get

$$
\left[\mathrm{Z}_{n}\right] \odot\left[\mathrm{Z}_{m}\right]:=\left\{\left[\mathrm{Z}_{n} \cap \mathrm{QZ}_{m} \mathrm{Q}^{-1}\right], \quad \mathrm{Q} \in \mathrm{O}(2)\right\}=\left\{\left[\mathrm{Z}_{n} \cap \mathrm{Z}_{m}\right]\right\}=\left\{\left[\mathrm{Z}_{d(n, m)}\right]\right\} .
$$

Now, $\mathrm{D}_{n}$ is generated by $\mathrm{Z}_{n}$ and the mirror symmetry $\sigma_{x}$ along the axis $(O x)$. Therefore

$$
\mathrm{D}_{n} \cap \mathrm{QZ}_{m} \mathrm{Q}^{-1}=\mathrm{D}_{n} \cap \mathrm{Z}_{m}=\mathrm{Z}_{n} \cap \mathrm{Z}_{m}
$$


and thus

$$
\left[\mathrm{D}_{n}\right] \odot\left[\mathrm{Z}_{m}\right]=\left\{\left[\mathrm{Z}_{d(n, m)}\right]\right\} .
$$

To compute the clips $\left[\mathrm{D}_{n}\right] \odot\left[\mathrm{D}_{m}\right]$, note that given $\mathrm{Q} \in \mathrm{O}(2)$, the subgroup $\mathrm{QD}_{m} \mathrm{Q}^{-1}$ is generated by $\mathrm{Z}_{m}$ and the mirror symmetry along the $\mathrm{Q}(O x)$ axis. We get therefore

$$
\left[\mathrm{D}_{n}\right] \odot\left[\mathrm{D}_{m}\right]=\left\{\left[\mathrm{Z}_{d(n, m)}\right],\left[\mathrm{D}_{d(n, m)}\right]\right\} .
$$

The other clips operations are obvious.

Using iterated clips operations, we get the following lemma.

Lemma 4.8. For $n \geq 1$ :

$$
\forall k \geq 1, \quad \odot^{k} \mathfrak{I}\left(\mathbb{K}^{n}\right):=\mathfrak{I}\left(\mathbb{K}^{n}\right) \odot\left(\odot^{k-1} \mathfrak{I}\left(\mathbb{K}^{n}\right)\right)=\left\{\left[\mathrm{Z}_{n}\right],\left[\mathrm{D}_{n}\right],[\mathrm{O}(2)]\right\}
$$

with $\odot{ }^{0}\left(\mathbb{K}^{n}\right):=\mathbb{K}^{n}$.

We have moreover:

Proposition 4.9. For $p \geq 2$ :

$$
\mathfrak{I}\left(\mathbb{K}^{2 p} \oplus \mathbb{K}^{2 p-2}\right)=\left\{\left[\mathrm{Z}_{2}\right],\left[\mathrm{D}_{2}\right],\left[\mathrm{D}_{2 p-2}\right],\left[\mathrm{D}_{2 p}\right],[\mathrm{O}(2)]\right\}
$$

Proof. From corollary 4.6 we have

$$
\mathfrak{I}\left(\mathbb{K}^{2 p} \oplus \mathbb{K}^{2 p-2}\right)=\mathfrak{I}\left(\mathbb{K}^{2 p}\right) \odot \mathfrak{I}\left(\mathbb{K}^{2 p-2}\right) .
$$

Now from theorem 4.1, $\mathfrak{I}\left(\mathbb{K}^{n}\right)=\left\{\left[\mathrm{D}_{n}\right],[\mathrm{O}(2)]\right\}(n \geq 0)$ and thus from table 8 we get:

\begin{tabular}{|c||c|c|}
\hline$\odot$ & {$\left[\mathrm{D}_{2 p}\right]$} & {$[\mathrm{O}(2)]$} \\
\hline \hline$\left[\mathrm{D}_{2 p-2}\right]$ & {$\left[\mathrm{Z}_{d(2 p, 2 p-2)}\right],\left[\mathrm{D}_{d(2 p, 2 p-2)}\right]$} & {$\left[\mathrm{D}_{2 p-2}\right]$} \\
\hline$[\mathrm{O}(2)]$ & {$\left[\mathrm{D}_{2 p}\right]$} & {$[\mathrm{O}(2)]$} \\
\hline
\end{tabular}

But $d(2 p, 2 p-2)=2$ for $p \geq 1$, which achieved the proof.

\subsection{Proof of the main result}

Recall first that given a constitutive tensor space $\mathbb{T}^{n}$, we necessarily have $\alpha_{n}=1$ and $\alpha_{k}=0$ for $k>n$, if $\mathbb{T}^{n}=\bigoplus_{k} \alpha_{k} \mathbb{K}^{k}$, because

$$
\mathbb{S}^{n} \subset \mathbb{T}^{n} \subset \mathbb{G}^{n}
$$

We will now determine sufficient conditions on the isotypic decomposition of $\mathbb{T}^{n}$ to have $\mathfrak{I}\left(\mathbb{T}^{n}\right)=\mathfrak{I}_{\text {max }}^{n}$. We will split the study according to $n$ is even or odd.

Proposition 4.10. Let $\mathbb{T}^{2 p}(p>0)$ be an even order tensor space which contains the irreducible representation $\mathbb{K}^{-1}$ in its harmonic decomposition. Then

$$
\mathfrak{I}\left(\mathbb{T}^{2 p}\right)=\mathfrak{I}_{\text {max }}^{2 p} .
$$

Proof. We can write

$$
\mathbb{T}^{2 p} \simeq \alpha_{-1} \mathbb{K}^{-1} \oplus \bigoplus_{k=0}^{p} \alpha_{2 k} \mathbb{K}^{2 k} \quad \text { with } \quad \alpha_{-1} \neq 0 .
$$

Since $\mathbb{S}^{2 p} \subset \mathbb{T}^{2 p}$ we have $\mathfrak{I}\left(\mathbb{S}^{2 p}\right) \subset \mathfrak{I}\left(\mathbb{T}^{2 p}\right)$. As a consequence of Lemma 2.5, we get

$$
\left\{\left[\mathrm{D}_{2 k}\right]\right\}_{1 \leq k \leq p} \cup\{[\mathrm{O}(2)]\} \subset \mathfrak{I}\left(\mathbb{T}^{2 p}\right) .
$$

Prepared using sagej.cls 
But since $\alpha_{-1} \neq 0$, we have

$$
\{[\mathrm{SO}(2)],[\mathrm{O}(2)]\} \odot\left(\left\{\left[\mathrm{D}_{2 k}\right]\right\}_{1 \leq k \leq p} \cup\{[\mathrm{O}(2)]\}\right) \subset \mathfrak{I}\left(\mathbb{T}^{2 p}\right)
$$

Now

$$
\begin{aligned}
\{[\mathrm{SO}(2)],[\mathrm{O}(2)]\} \odot\left(\left\{\left[\mathrm{D}_{2 k}\right]\right\}_{1 \leq k \leq p} \cup\{[\mathrm{O}(2)]\}\right) & =\left\{\left[\mathrm{Z}_{2 k}\right],\left[\mathrm{D}_{2 k}\right]\right\}_{1 \leq k \leq p} \cup\{[\mathrm{SO}(2)],[\mathrm{O}(2)]\} \\
& =\mathfrak{I}_{\max }^{2 p} .
\end{aligned}
$$

Thus $\mathfrak{I}\left(\mathbb{S}^{2 p}\right)=\mathfrak{I}_{\max }^{2 p} \subset \mathfrak{I}\left(\mathbb{T}^{2 p}\right) \subset \mathfrak{I}_{\max }^{2 p}$, which achieves the proof.

In fact there is no other way to obtain a complete set of classes. Nevertheless, in the odd order case we have:

Proposition 4.11. Let $\mathbb{T}^{2 q+1}$ be an odd-order tensor space such that:

$$
\mathbb{T}^{2 q+1} \simeq \bigoplus_{k=0}^{q} \alpha_{2 k+1} \mathbb{K}^{2 k+1} \quad \text { where } \quad \alpha_{2 k+1} \geq 2, \quad \text { for } k<q .
$$

Then

$$
\mathfrak{I}\left(\mathbb{T}^{2 q+1}\right)=\mathfrak{I}_{\max }^{2 q+1} \backslash\left\{\left[\mathrm{Z}_{2 q+1}\right]\right\} .
$$

Proof. Since $\mathbb{S}^{2 q+1} \subset \mathbb{T}^{2 q+1}$, we have $\mathfrak{I}\left(\mathbb{S}^{2 q+1}\right) \subset \mathfrak{I}\left(\mathbb{T}^{2 q+1}\right)$ and thus by Lemma 2.5 , we get

$$
\left\{\left[\mathrm{D}_{2 k+1}\right]\right\}_{0 \leq k \leq q} \cup\{[\mathrm{O}(2)]\} \subset \mathfrak{I}\left(\mathbb{T}^{2 q+1}\right) .
$$

For $k<q$ we have $\alpha_{2 k+1} \geq 2$ and $\alpha_{2 q+1}=1$. Now

$$
\mathfrak{I}\left(\alpha_{2 k+1} \mathbb{K}^{2 k+1}\right)=\odot^{\alpha_{2 k+1}}\left(\mathbb{K}^{2 k+1}\right)=\left\{\left[\mathrm{Z}_{2 k+1}\right],\left[\mathrm{D}_{2 k+1}\right],[\mathrm{O}(2)]\right\} .
$$

and for the last irreducible representation

$$
\mathfrak{I}\left(\mathbb{K}^{2 q+1}\right)=\left\{\left[\mathrm{D}_{2 q+1}\right],[\mathrm{O}(2)]\right\} .
$$

which show all isotropy classes in $\mathfrak{I}_{\max }^{2 q+1}$ appear but $Z_{2 q+1}$, hence

$$
\mathfrak{I}\left(\mathbb{T}^{2 q+1}\right)=\mathfrak{I}_{\max }^{2 q+1} \backslash\left\{\left[\mathrm{Z}_{2 q+1}\right]\right\} .
$$

which concludes the proof.

These first two results and the harmonic decompositions of $\mathbb{G}^{2 p}$ and $\mathbb{G}^{2 p+1}$ given by Lemma 3.9 directly lead to the following lemma.

Lemma 4.12. We have:

$$
\left\{\begin{array}{l}
\mathfrak{I}\left(\mathbb{G}^{2 p}\right)=\mathfrak{I}_{\max }^{2 p} \\
\mathfrak{I}\left(\mathbb{G}^{2 q+1}\right)=\mathfrak{I}_{\max }^{2 q+1} \backslash\left\{\left[\mathrm{Z}_{2 q+1}\right]\right\}
\end{array} .\right.
$$

Let us now observe that given two integers $m_{1}, m_{2}>0$, we get the following:

- If $m_{1}$ and $m_{2}$ have the same parity, the harmonic decomposition of $\mathbb{S}^{m_{1}} \otimes \mathbb{S}^{m_{2}}$ involves a tensor product of two spaces of same order. As a consequence of the Clebsch-Gordan formula (Lemma 3.4), there is a term $\mathbb{K}^{-1}$ in the decomposition. Thus, we can apply Proposition 4.10 on $\mathbb{S}^{m_{1}} \otimes \mathbb{S}^{m_{2}}$ to achieve the proof;

- If $m_{1}$ and $m_{2}$ have different parity (i.e. $m_{1}=2 p$ and $m_{2}=2 q+1$ ) a direct induction on $p+q$ leads to the harmonic decomposition

$$
\mathbb{S}^{2 p} \otimes \mathbb{S}^{2 q+1}=\bigoplus_{k=0}^{p+q} \alpha_{2 k+1} \mathbb{K}^{2 k+1} \quad \text { with } \quad \alpha_{2 k+1} \geq 2, \quad \text { for } \quad k<p+q .
$$

Thus we can apply Proposition 4.11 on $\mathbb{S}^{m_{1}} \otimes \mathbb{S}^{m_{2}}$. 
Finally we have:

Lemma 4.13. Let $m_{1}, m_{2}>0$. We have:

$$
\mathfrak{I}\left(\mathbb{S}^{m_{1}} \otimes \mathbb{S}^{m_{2}}\right)=\left\{\begin{array}{l}
\mathfrak{I}_{m a x}^{m_{1}+m_{2}}, \quad \text { if } m_{1}, m_{2} \text { have the same parity, } \\
\mathfrak{I}_{\max }^{m_{1}+m_{2}} \backslash\left\{\left[\mathrm{Z}_{m_{1}+m_{2}}\right]\right\}, \quad \text { if } m_{1}, m_{2} \text { have different parity. }
\end{array} .\right.
$$

In the case of the symmetric tensor space $S^{2}\left(\mathbb{S}^{n}\right)$ :

Lemma 4.14. For any integer $n \geq 2$, we have:

$$
\mathfrak{I}\left(S^{2}\left(\mathbb{S}^{n}\right)\right)=\mathfrak{I}_{\max }^{2 n} \backslash\left\{\left[\mathrm{Z}_{2 n}\right],\left[\mathrm{Z}_{2 n-2}\right]\right\} .
$$

Proof. Using the harmonic decomposition of $S^{2}\left(\mathbb{S}^{n}\right)$ given in Lemma 3.7, all irreducible representations $\mathbb{K}^{2 k}(k<n-1)$ come with multiplicity $\alpha_{2 k} \geq 2$ and thus by Lemma 4.8 we have

$$
\mathfrak{I}\left(\alpha_{2 k} \mathbb{K}^{2 k}\right)=\odot^{\alpha_{2 k}}\left(\mathbb{K}^{2 k}\right)=\left\{\left[\mathrm{Z}_{2 k}\right],\left[\mathrm{D}_{2 k}\right],[\mathrm{O}(2)]\right\} .
$$

Now $\mathbb{K}^{2 n} \oplus \mathbb{K}^{2 n-2}$ is also present in the isotypic decomposition of $S^{2}\left(\mathbb{S}^{n}\right)$, and by Proposition 4.9

$$
\mathfrak{I}\left(\mathbb{K}^{2 n} \oplus \mathbb{K}^{2 n-2}\right)=\left\{\left[\mathrm{Z}_{2}\right],\left[\mathrm{D}_{2}\right],\left[\mathrm{D}_{2 n-2}\right],\left[\mathrm{D}_{2 n}\right],[\mathrm{O}(2)]\right\} .
$$

Applying the clips operator to these two sets, we get finally

$$
\mathfrak{I}\left(S^{2}\left(\mathbb{S}^{n}\right)\right)=\mathfrak{I}_{\max }^{2 n} \backslash\left\{\left[\mathrm{Z}_{2 n}\right],\left[\mathrm{Z}_{2 n-2}\right]\right\} .
$$

Proof of Theorem 2.4. When $\mathbb{T}^{n}=\mathbb{G}^{n}$ or $\mathbb{T}^{n}=S^{2}\left(\mathbb{S}^{n}\right)$, the theorem is a direct consequence of Lemma 4.12 and Lemma 4.14. We still need to consider the case $S^{2}\left(\mathbb{T}^{n}\right)$ where $\mathbb{T}^{n} \supsetneq \mathbb{S}^{n}$ and the case $\mathbb{T}^{m_{1}} \otimes \mathbb{T}^{m_{2}}$ (coupling constitutive laws).

If $\mathbb{T}^{n} \supsetneq \mathbb{S}^{n}$, the harmonic decomposition of $\mathbb{T}^{n}$ contains necessarily a factor $\mathbb{K}^{k} \oplus \mathbb{K}^{k}$ and thus $S^{2}\left(\mathbb{T}^{n}\right)$ contains a factor

$$
S^{2}\left(\mathbb{K}^{k} \oplus \mathbb{K}^{k}\right) \simeq S^{2}\left(\mathbb{K}^{k}\right) \oplus S^{2}\left(\mathbb{K}^{k}\right) \oplus\left(\mathbb{K}^{k} \otimes \mathbb{K}^{k}\right) .
$$

Thus, the factor $\mathbb{K}^{-1}$ is present in the harmonic decomposition of $S^{2}\left(\mathbb{T}^{n}\right)$ and due to proposition 4.10, we have then $\mathfrak{I}\left(S^{2}\left(\mathbb{T}^{n}\right)\right)=\mathfrak{I}_{\max }^{2 n}$.

In the case $\mathbb{T}^{m_{1}} \otimes \mathbb{T}^{m_{2}}$, we always have $\mathbb{S}^{m_{1}} \otimes \mathbb{S}^{m_{2}} \subset \mathbb{T}^{m_{1}} \otimes \mathbb{T}^{m_{2}}$.

- if $m_{1}$ and $m_{2}$ have the same parity, the conclusion follows from Lemma 4.13;

- if $m_{1}$ and $m_{2}$ don't have the same parity we have

$$
\mathfrak{I}_{\max }^{m_{1}+m_{2}} \backslash\left\{\left[\mathrm{Z}_{m_{1}+m_{2}}\right]\right\} \subset \mathfrak{I}\left(\mathbb{T}^{m_{1}} \otimes \mathbb{T}^{m_{2}}\right),
$$

by Lemma 4.13. Besides, the factor $\mathbb{K}^{m_{1}+m_{2}}$ occurs in the harmonic decomposition of $\mathbb{T}^{m_{1}} \otimes \mathbb{T}^{m_{2}}$ with multiplicity one and since

$$
\mathfrak{I}\left(\mathbb{K}^{m_{1}+m_{2}}\right)=\left\{\left[\mathrm{D}_{m_{1}+m_{2}}\right],[\mathrm{O}(2)]\right\}
$$

by Theorem 4.1, we get

$$
\mathfrak{I}\left(\mathbb{T}^{m_{1}} \otimes \mathbb{T}^{m_{2}}\right) \subset \mathfrak{I}_{\max }^{m_{1}+m_{2}} \backslash\left\{\left[\mathrm{Z}_{m_{1}+m_{2}}\right]\right\}
$$

which achieves the proof.

\section{Conclusion}

In the present paper, the general problem of finding the symmetry classes of $2 \mathrm{D}$ constitutive tensor spaces has been solved. To tackle this question, numerous tools from group representation theory have been used. We believe that these methods could find applications to other problems. In a forthcoming paper, the harmonic decomposition will be used to generate bases of polynomial invariants, i.e. integrity bases, for general constitutive tensor spaces in 2D. The knowledge of an integrity basis has many practical interests ranging from the labelling of materials to the formulation of non-linear constitutive laws. 


\section{Acknowledgements}

N. Auffray and B. Kolev would like to gratefully acknowledge the Région Pays de la Loire and the pole $E M C 2$ for their financial support.

\section{Declaration of conflicting interests}

None declared.

\section{A Elements of representation theory}

\section{A.1 Group representations}

Let $G$ denote a compact group and $\mathbb{V}$ a finite dimensional $\mathbb{R}$-vector space. A linear representation $(\mathbb{V}, \rho)$ of $G$ is a group morphism from $G$ to the group $\mathrm{GL}(\mathbb{V})$ of linear invertible endomorphisms of $\mathbb{V}:$

$$
\rho: G \longrightarrow \mathrm{GL}(\mathbb{V}) .
$$

Thus, for each element $g \in G$, we have an invertible linear mapping $\rho(g)$ of $\mathbb{V}$ and for each $\mathbf{v} \in \mathbb{V}$ we write

$$
g \cdot \mathbf{v}:=\rho(g)(\mathbf{v})
$$

Two representations $\left(\rho_{1}, \mathbb{V}_{1}\right)$ and $\left(\rho_{2}, \mathbb{V}_{2}\right)$ of $G$ are said to be equivalent if there exists an isomorphism $\varphi$ such that the following diagram is commutative

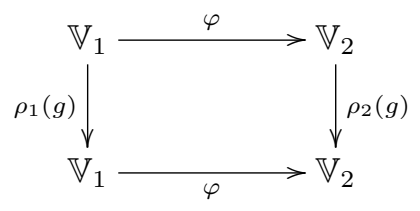

The character of a representation $(\mathbb{V}, \rho)$ of $G$ is the real value function

$$
\chi_{\rho}(g):=\operatorname{tr}(\rho(g))
$$

Note that two equivalent representations $\left(\rho_{1}, \mathbb{V}_{1}\right)$ and $\left(\rho_{2}, \mathbb{V}_{2}\right)$ of $G$ have the same character. Furthermore, for two representations $(\mathbb{V}, \rho)$ and $\left(\mathbb{V}^{\prime}, \rho^{\prime}\right)$ of $G$, the characters of the induced representations $\left(\mathbb{V} \oplus \mathbb{V}^{\prime}, \rho \oplus \rho^{\prime}\right)$ and $\left(\mathbb{V} \otimes \mathbb{V}^{\prime}, \rho \otimes \rho^{\prime}\right)$ are given by:

$$
\chi_{\rho \oplus \rho^{\prime}}=\chi_{\rho}+\chi_{\rho^{\prime}} \quad ; \quad \chi_{\rho \otimes \rho^{\prime}}=\chi_{\rho} \chi_{\rho^{\prime}}
$$

\section{A.2 Decomposition into irreducible representations}

A subspace $\mathbb{W}$ of a linear representation $(\mathbb{V}, \rho)$ of a group $G$ is stable if

$$
g \cdot \mathbf{w} \in \mathbb{W}, \quad \forall \mathbf{w} \in \mathbb{W}, \quad \forall g \in G .
$$

The linear representation $(\mathbb{V}, \rho)$ is irreducible if $\{0\}$ and $\mathbb{V}$ are the only stable subspaces of $\mathbb{V}$. Due to Peter-Weyl's theorem [33], we know that every finite dimensional representation $\mathbb{V}$ of a compact group $G$ can be split into a direct sum of finite dimensional irreducible representations

$$
\mathbb{V} \simeq \mathbb{V}_{k_{1}} \oplus \mathbb{V}_{k_{2}} \oplus \ldots \oplus \mathbb{V}_{k_{n}}
$$

where, for each $k_{i},\left(\mathbb{V}_{k_{i}}, \rho_{k_{i}}\right)$ is an irreducible representation of $G$, and $\simeq$ indicates a $G$-equivariant isomorphism. We consider also the isotypic decomposition, in which equivalent factors are group together

$$
\mathbb{V} \simeq \alpha_{1} \mathbb{V}_{1} \oplus \alpha_{2} \mathbb{V}_{2} \oplus \ldots \oplus \alpha_{n} \mathbb{V}_{n}, \quad \alpha_{i} \mathbb{V}_{i}:=\bigoplus_{l=1}^{\alpha_{i}} \mathbb{V}_{i}
$$

When applied to the special case of a finite dimensional representation of the orthogonal group $\mathrm{O}(2)$ the isotypic decomposition is often referred to as the harmonic decomposition $[9,25,17,19]$. 


\section{A.3 Character's formula}

The characters $\chi_{\rho_{i}}$ of the irreducible representations $\left(\mathbb{V}_{i}, \rho_{i}\right)$ constitute an orthonormal system for the inner product

$$
\left\langle f_{1}, f_{2}\right\rangle:=\int_{G} f_{1}(g) f_{2}(g) d \mu(g) .
$$

where $\mu$ is the Haar mesure on $G$. Thus, using the rule (A.1), the coefficient $\alpha_{i}$ of the irreducible representation $\left(\mathbb{V}_{i}, \rho_{i}\right)$ in (A.2) is given by:

$$
\alpha_{i}=\left\langle\chi_{\rho}, \chi_{\rho_{i}}\right\rangle
$$

The characters of the irreducible representations $\left(\mathbb{K}^{n}, \rho_{n}\right)(n=-1,0, \ldots)$ of $\mathrm{O}(2)$ has the following explicit expressions

$$
\chi_{\rho_{n}}\left(r_{\theta}\right)=2 \cos (n \theta), \quad \chi_{\rho_{n}}\left(\sigma_{x} r_{\theta}\right)=0, \quad(n \geq 1)
$$

and

$$
\chi_{\rho_{0}} \equiv 1, \quad \chi_{\rho_{-1}}\left(r_{\theta}\right)=1, \quad \chi_{\rho_{-1}}\left(\sigma_{x} r_{\theta}\right)=-1 .
$$

For $\mathrm{O}(2)$, the inner product (A.3) is explicitly given by

$$
\left\langle f_{1}, f_{2}\right\rangle:=\frac{1}{4 \pi} \int_{0}^{2 \pi} f_{1}\left(r_{\theta}\right) f_{2}\left(r_{\theta}\right) d \theta+\frac{1}{4 \pi} \int_{0}^{2 \pi} f_{1}\left(\sigma_{x} r_{\theta}\right) f_{2}\left(\sigma_{x} r_{\theta}\right) d \theta .
$$

\section{Notes}

1. It has to be noted that $Z_{2}^{\sigma_{x}}$ and $Z_{2}$ are isomorphic as groups but are not conjugate.

2. The name for the different bi-dimensional symmetry systems are borrowed from [39].

3. There is some ambiguity in the literature on what is the proper generalisation of the classical notion of deviator to higher-order tensors. For some authors [34, 27] it means tensors that contain no scalar part, for others [25] it means traceless symmetric higher-order tensors. These definitions coincide for secondorder tensors, but differ for higher-order tensors. We prefer the second definition since then deviators correspond to irreducible representations of the orthogonal group.

\section{References}

[1] J. M. Ansemil and K. Floret. The symmetric tensor product of a direct sum of locally convex spaces. Stud. Math., 129(3):285-295, 1998.

[2] M. A. Armstrong. Groups and symmetry. Springer, 1983.

[3] N. Auffray, R. Bouchet, and Y. Bréchet. Derivation of anisotropic matrix for bi-dimensional strain-gradient elasticity behavior. Int. J. Solids Struct., 46(2):440-454, 2009.

[4] N. Auffray, J. Dirrenberger, and G. Rosi. A complete description of bi-dimensional anisotropic strain-gradient elasticity. Int. J. Solids Struct., 69-70:195-206, 2015.

[5] N. Auffray, B. Kolev, and M. Petitot. On anisotropic polynomial relations for the elasticity tensor. J. Elasticity, 115:77-103, 2014.

[6] N. Auffray, B. Kolev, and M. Petitot. A complete integrity basis for the elasticity tensor. To be submitted, -:-, 2016.

[7] N. Auffray and P. Ropars. Invariant-based reconstruction of bidimensionnal elasticity tensors. Int. J. Solids Struct., -:(On-line First), 2016.

[8] A. Bacigalupo and L. Gambarotta. Homogenization of periodic hexa-and tetrachiral cellular solids. Compos. Struct., 116:461-476, 2014.

[9] G. Backus. A geometrical picture of anisotropic elastic tensors. Rev. Geophys., 8(3):633-671, 1970. 
[10] A. Blinowski, J. Ostrowska-Maciejewska, and J. Rychlewski. Two-dimensional Hooke's tensors - isotropic decomposition, effective symmetry criteria. Arch. Mech., 48:325-345, 1996.

[11] J.-P. Boehler, A. A. Kirillov, Jr., and E. T. Onat. On the polynomial invariants of the elasticity tensor. J. Elasticity, 34(2):97-110, 1994.

[12] G. Bredon. Introduction to compact transformation groups. Academic Press, New York, 1972. Pure and Applied Mathematics, Vol. 46.

[13] Y. Chen, X. Liu, G. Hu, and Q. Sun. Micropolar continuum modelling of bi-dimensional tetrachiral lattices. Proc. R. Soc. A, 470(2165):20130734, 2014.

[14] G. de Saxcé and C. Vallée. Invariant measures of the lack of symmetry with respect to the symmetry groups of 2"d" elasticity tensors. J. Elasticity, 111:21-39, 2013.

[15] B. Desmorat and P. Vannucci. An alternative to the Kelvin decomposition for plane anisotropic elasticity. Math. Meth. Appl. Sci., 38(1):164-175, 2015.

[16] S. Forest. Mechanics of generalized continua: construction by homogenizaton. J. Phys. IV, 8(PR4):Pr4-39, 1998.

[17] S. Forte and M. Vianello. Symmetry classes for elasticity tensors. J. Elasticity, 43(2):81-108, 1996.

[18] S. Forte and M. Vianello. Symmetry classes and harmonic decomposition for photoelasticity tensors. Int. J. Eng. Sci., 35(14):1317 - 1326, 1997.

[19] S. Forte and M. Vianello. A unified approach to invariants of plane elasticity tensors. Meccanica, 49(9):2001-2012, 2014.

[20] M. L. François. A damage model based on Kelvin eigentensors and Curie principle. Mech. Mater., 44:23-34, 2012.

[21] G. Geymonat and T. Weller. Symmetry classes of piezoelectric solids. C.R. Mathématique, $335(10): 847-852,2002$.

[22] M. Golubitsky, I. Stewart, and D. G. Schaeffer. Singularities and groups in bifurcation theory. Vol. II, volume 69 of Applied Mathematical Sciences. Springer-Verlag, New York, 1988.

[23] Q. C. He and Q. S. Zheng. On the symmetries of 2"d" elastic and hyperelastic tensors. J. Elasticity, 43:203-225, 1996.

[24] B. Herman. Some theorems of the theory of anisotropic media. Comptes Rendus (Doklady) Acad. Sci. URSS, 48(2):89-92, 1945.

[25] J. Jerphagnon, D. Chemla, and R. Bonneville. The description of the physical properties of condensed matter using irreducible tensors. Adv. Phys., 27:609-650, 1978.

[26] H. Le Quang and Q.-C. He. The number and types of all possible rotational symmetries for flexoelectric tensors. Proc. R. Soc. A, 467(2132):2369-2386, 2011.

[27] R. Mindlin. Micro-structure in linear elasticity. Arch. Rational Mech. Anal., 16(1), 1964.

[28] R. Mindlin and N. Eshel. On first strain-gradient theories in linear elasticity. Int. J. Solids Struct., 4(1):109-124, 1968.

[29] M. Olive and N. Auffray. Symmetry classes for even-order tensors. Mathematics and Mechanics of Complex Systems, 1:177-210, 2013.

[30] M. Olive and N. Auffray. Symmetry classes for odd-order tensors. ZAMM Z. Angew. Math. Mech., 94:421-447, 2014. 
[31] P. Olver. Classical invariant theory, volume 44 of London Mathematical Society Student Texts. Cambridge University Press, Cambridge, 1999.

[32] G. Rosi and N. Auffray. Anisotropic and dispersive wave propagation within strain-gradient framework. Wave Motion, In Press:-, 2016.

[33] S. Sternberg. Group theory and physics. Cambridge University Press, Cambridge, 1994.

[34] R. A. Toupin. Elastic materials with couple-stresses. Arch. Ration. Mech. Anal., 11:385-414, 1962.

[35] D. K. Trinh, R. Janicke, N. Auffray, S. Diebels, and S. Forest. Evaluation of generalized continuum substitution models for heterogeneous materials. Int. J. Multiscale Com., 10(6), 2012 .

[36] P. Vannucci. The polar analysis of a third order piezoelectricity-like plane tensor. Int. J. Solids Struct., 44:7803-7815, 2007.

[37] M. Vianello. An integrity basis for plane elasticity tensors. Arch. Mech., 49:197-208, 1997.

[38] A. Vincenti, P. Vannucci, and M. R. Ahmadian. Optimization of laminated composites by using genetic algorithm and the polar description of plane anisotropy. Mech. Adv. Mater. Struc., 20:242-255, 2013.

[39] Q.-S. Zheng and J.-P. Boehler. The description, classification, and reality of material and physical symmetries. Acta Mech., 102(1-4):73-89, 1994. 